\title{
JEAN-PIERRE KAHANE Sur trois notes de Stieltjes relatives aux séries de Dirichlet
}

Annales de la faculté des sciences de Toulouse $6^{e}$ série, tome S5 (1996), p. 33-56

<http://www.numdam.org/item?id=AFST_1996_6_S5_33_0>

(C) Université Paul Sabatier, 1996, tous droits réservés.

L'accès aux archives de la revue «Annales de la faculté des sciences de Toulouse » (http://picard.ups-tlse.fr/ annales/) implique l'accord avec les conditions générales d'utilisation (http://www.numdam.org/conditions). Toute utilisation commerciale ou impression systématique est constitutive d'une infraction pénale. Toute copie ou impression de ce fichier doit contenir la présente mention de copyright.

\section{NumDam}

Article numérisé dans le cadre du programme

Numérisation de documents anciens mathématiques

http://www.numdam.org/ 


\title{
Sur trois notes de Stieltjes relatives aux séries de Dirichlet
}

\author{
Jean-Pierre Kahane ${ }^{(1)}$
}

En 1885, Stieltjes crut avoir démontré l'hypothèse de Riemann et le théorème des nombres premiers. C'est l'objet de deux notes aux Comptes rendus qui ont eu une postérité remarquable. D'abord, elles ont stimulé toutes les recherches ultérieures sur les zéros de la fonction dzèta et sur la répartition des nombres premiers. Ensuite, elles ont introduit un bon outil, qu'on appelle dans les traités de probabilité le lemme de Kronecker. Enfin, elles ont inauguré un nouveau sujet, la convergence des produits de séries de Dirichlet, auquel Stieltjes lui-même est revenu en 1887, qui a donné naissance à beaucoup de travaux et dont l'intérêt ne me parait pas épuisé.

Nous allons d'abord indiquer rapidement le contenu de ces trois notes. Puis, nous exposerons une méthode simple, reposant sur l'analyse de Fourier, pour démontrer le théorème des nombres premiers et l'étendre aux nombres premiers généralisés de Beurling; ce sera notre contribution au centenaire de ce théorème. Le théorème 1 de la seconde note nous donnera l'occasion d'évoquer l'histoire du lemme de Kronecker. Tout le reste de l'article sera consacré à la postérité du théorème 2 de la seconde note, sur la convergence des produits de séries de Dirichlet, et aux travaux que j'ai en cours avec Hervé Queffélec. À la suite de Stieltjes, ce sujet a inspiré Cahen, Landau et Bohr. Récemment, Delange et Tenenbaum y sont revenus. Nous montrerons comment il se relie à des questions sur l'ordre et sur la sommabilité des séries de Dirichlet, qui ont intéressé Bohr depuis sa thèse jusqu'à la fin de sa vie, et sur lesquelles quelques progrès ont été réalisés récemment.

(1) Université de Paris-Sud, Mathématique, Bât. 425, F-91405 Orsay Cedex (France) 


\section{Table des matières}

1. Les trois notes de Stieltjes.

2. Le théorème des nombres premiers. Une preuve par analyse de Fourier. Un théorème et un problème sur les nombres premiers généralisés.

3. Intermède historique. La loi des grands nombres, le lemme de Kronecker et le théorème 1 de la deuxième note de Stieltjes.

4. Le théorème 2 de la deuxième note et sa postérité : convergence des séries produits.

5. La fonction de Lindelöf et la méthode de Bohr. Son extension grâce aux catégories de Baire. Résultats optimaux sur la convergence d'une série produit de séries de Dirichlet.

6. Ordre et sommabilité. Théorème de Cesàro. Théorème de Bohr. Définitions de Marcel Riesz. Problèmes et conjectures.

7. La sommabilité d'une série produit de séries de Dirichlet.

Références.

\section{Les trois notes de Stieltjes}

"Sur une fonction uniforme" est le titre d'une courte note aux Comptes rendus de l'Académie des sciences, présentée par Charles Hermite le 13 juillet 1885 [S1]. Il s'agit de la fonction

$$
\zeta(s)=\sum_{1}^{\infty} \frac{1}{n^{s}}, \quad s=\sigma+i t,
$$

définie comme somme de la série pour $\sigma>1$ et par prolongement analytique en une fonction méromorphe dans tout le plan des $s$. Riemann qui avait établi ce prolongement, au moyen de l'équation fonctionnelle qui lie $\zeta(1-s)$ à $\zeta(s)$, avait conjecturé que tous les zéros de $\zeta(s)$ se trouvent sur la droite $\sigma=1 / 2$. Pour montrer cela, il suffit de prouver que $\zeta(s)$ n'admet aucun zéro dans le demi-plan $\sigma>1 / 2$. C'est ce que crut faire Stieltjes en établissant que la série de Dirichlet de $1 / \zeta(s)$.

$$
\begin{aligned}
\sum_{1}^{\infty} \frac{\mu(n)}{n^{s}}( & \left.=\prod_{p \text { premier }}\left(1-\frac{1}{p^{s}}\right)\right) \\
& -34-
\end{aligned}
$$


converge pour $\sigma>1 / 2$. Il annonça dans sa note une démonstration, qui fût très attendue de ses contemporains et ne vit jamais le jour. Sur les raisons possibles de cette erreur de Stieltjes, et sur les développements récents de l'étude de la fonction

$$
M(x)=\sum_{n<x} \mu(n)
$$

on consultera avec profit l'article de H. J. J. te Riele dans la dernière édition des œuvres de Stieltjes [S4].

La note aux Comptes rendus qui suit ( 3 août 1885) est intitulée "Sur une loi asymptotique dans la théorie des nombres". Supposant acquis le résultat de la premier note, Stieltjes démontre la formule

$$
\theta(x)\left(=\sum_{p<x} \log p\right)=x+o\left(x^{3 / 4+\varepsilon}\right) \quad(x \rightarrow \infty) .
$$

La méthode est ingénieuse, et nous y reviendrons. Selon un théorème de Dirichlet, le nombre $d(n)$ de diviseurs de $n$ vérifie

$$
\sum_{m=1}^{n}(d(m)-\log m-2 C)=\mathrm{O}(\sqrt{n}),
$$

$C$ étant la constante d'Euler, donc la série

$$
\sum_{1}^{\infty} \frac{d(n)-\log n-2 C}{n^{s}}\left(=\zeta^{2}(s)+\zeta^{\prime}(s)-2 C \zeta(s)\right)
$$

converge pour $\sigma>1 / 2$. Il en est de même, croit Stieltjes, pour la série

$$
\sum_{1}^{\infty} \frac{\mu(n)}{n^{s}}\left(=\frac{1}{\zeta(s)}\right) .
$$

En multipliant les deux séries (A) et (B), on obtient

$$
\sum_{1}^{\infty} \frac{1-\Lambda(n)}{n^{s}}-2 C\left(=\zeta(s)+\frac{\zeta^{\prime}(s)}{\zeta(s)}-2 C\right) .
$$

Or, si les deux séries (A) et (B) convergent pour $\sigma>1 / 2$, la série produit (C) converge pour $\sigma>3 / 4$; c'est une conséquence du théorème 2 de la 
note, sur la multiplication des séries de Dirichlet. Il en résulte, d'après le théorème 1 de la note, que

$$
\sum_{n<x}(1-\Lambda(n))=\mathrm{o}\left(x^{3 / 4+\varepsilon}\right) \quad(x-\infty)
$$

d'où, facilement,

$$
\theta(x)=x+o\left(x^{3 / 4+\varepsilon}\right) \quad(x-\infty) .
$$

Comme corollaire (en fait, c'est un corollaire de $\theta(x) \sim x$ ) Stieltjes signale que, quel que soit $h>0$, le nombre de nombres premiers situés entre $x$ et $x(1+h)$ tend vers l'infini avec $x$ [S2].

La note "Sur la multiplication de deux séries" fut publiée dans les Nouvelles annales de mathématiques en 1887 [S3]. Après avoir remarqué que, pour deux séries absolument convergentes $\sum u_{\alpha}$ et $\sum v_{\beta}$. de sommes $s$ et $t$, la série produit $\sum u_{\alpha} v_{\beta}$, écrite dans un ordre quelconque, est absolument convergente et a pour somme st, Stieltjes considère le cas où la première série est convergente et la seconde absolument convergente. Alors la série produit est convergente et a pour somme le produit des sommes, à la seule condition de sommer la série produit sous la forme

$$
\lim _{n \rightarrow \infty} \sum_{(\alpha, \beta) \in D_{n}} u_{\alpha} v_{\beta}
$$

où des $D_{n}$ sont des parties croissante de $\mathbb{N}^{2}$, tendant vers $\mathbb{N}^{2}$ et dont l'intersection avec chaque horizontale ( $\beta$ fixé) est une section commençante de $\mathbb{N}\left(\alpha \leq \varphi_{n}(\beta)\right)$. Par exemple, on peut prendre $\alpha+\beta \leq n$ (cas du produit de séries de Taylor) ou $\alpha \beta \leq n$ (cas du produit de séries de Dirichlet). En ce qui concerne les séries de Dirichlet. Stieltjes ajoute, sans démonstration, deux propositions :

1) si deux séries de Dirichlet convergent en $s=a$, et convergent absolument en $s=\alpha+\beta$ et $\alpha+\gamma$ respectivement, la série produit converge en $s=\alpha+\beta \gamma /(\beta+\gamma)$;

2) si deux séries de Dirichlet convergent en $s=\alpha$, la série produit converge en $s=a+1 / 2$.

Le théorème 2 de la seconde note de 1885 est la première de ces propositions, dans le cas $\beta=$ ?. 


\section{Le théorème des nombres premiers.} Une preuve par analyse de Fourier.

\section{Un théorème et un problème sur les nombres premiers généralisés}

En 1896, Hadamard et de La Vallée Poussin établirent indépendamment que la fonction $\zeta(s)$ ne s'annule pas sur la droite $\sigma=1$, et en tirèrent le théorème des nombres premiers ([Ha], [VP]) :

$$
\pi(x) \sim \frac{x}{\log x} .
$$

Depuis un siècle les démonstrations se sont multipliées. Bornons-nous à quelques repères. Le livre d'Ingham sur la distribution des nombres premiers fait excellemment le point en 1932, et l'avant-propos écrit par Vaughan pour l'édition de 1990 est une mise au point actuelle [I]. En 1932 également, parait l'étude de Wiener sur les théorèmes taubériens, qui montre bien le lien entre analyse de Fourier et répartition des nombres premiers [W]. En 1937, Beurling introduit les nombres premiers généralisés [Be]. En 1949, Erdös et Selberg donnent la première preuve élémentaire du théorème (ne reposant pas sur la théorie des fonctions d'une variable complexe) ([E],[Se]). Une preuve élémentaire simplifiée est due à Wirsing et à Bang ([Ba], [Wi]) en 1962 et 1963. En 1972, Helson donne le principe d'une preuve rapide, fondée sur les séries de Dirichlet et la formule de Plancherel, qui est exposée dans le livre d'Ellison et Mendès-France sur les nombres premiers ([He], [EM]). En 1980, Newman publie une preuve analytique très simple [N]. En 1984, Daboussi donne en trois pages une preuve élémentaire complète [Da].

Voici la méthode d'analyse de Fourier que j'ai indiquée tout récemment dans la Gazette des mathématiciens ( ${ }^{\circ} 67$, janvier 1996). Je prendrai ici pour cadre les nombres premiers généralisés de Beurling, dont l'ensemble sera désigné par $P$, et les entiers généralisés correspondants, dont l'ensemble sera désigné par $N$. La fonction dzèta est ici

$$
\zeta(s)=\prod_{p \in P}\left(1-\frac{1}{p^{s}}\right)^{-1}=\sum_{n \in N} \frac{1}{n^{s}} \quad(\sigma>1) .
$$

On désigne respectivement par $\varpi(x)$ et $\nu(x)$ les fonctions de comptage de $P$ et de $N$. Nous ferons l'hypothèse que, pour un $\mathrm{D}>0$ et un $\varepsilon>0$, on a

$$
\nu(x)=D x+\mathrm{O}\left(x^{1-\varepsilon}\right) \quad(x \rightarrow \infty) .
$$


La conclusion sera

$$
\varpi(x) \sim \frac{x}{\log x} \quad(x \rightarrow \infty)
$$

La formule

$$
\begin{aligned}
\zeta(s) & =\frac{D}{s-1}+\int_{1}^{\infty} x^{-s} \mathrm{~d}(\nu(x)-x) \\
& =\frac{D}{s-1}+\int_{1}^{\infty} s x^{-s-1}(\nu(x)-x) \mathrm{d} x
\end{aligned}
$$

permet de prolonger $\zeta(s)$ en une fonction méromorphe dans le demi-plan $\sigma>1-\varepsilon$, avec un seul pôle, simple et de résidu $D$, au point $s=1$.

Considérons

$$
Z(s)=\exp \sum_{p \in P} \frac{1}{p^{s}} \quad(\sigma>1) .
$$

Comme

$$
\log (1-u)+u=\mathrm{O}\left(|u|^{2}\right) \quad(u \text { complexe } \rightarrow 0)
$$

et que la série $\sum_{p \in P} 1 / p^{2 \sigma}$ converge pour $\sigma>1 / 2$, la fonction $Z(s) / \zeta(s)$ se prolonge en une fonction holomorphe et sans zéro dans le demi-plan $\sigma>1 / 2$, donc $Z(s)$ est holomorphe pour $\sigma>1$, méromorphe pour $\sigma>\sup (1 / 2,1-\varepsilon)$ avec les mémes pôles et zéros que $\zeta(s)$ dans ce demiplan.

Posons, pour $t$ réel et $\varepsilon>0$,

$$
\ell_{\varepsilon}(t)=\sum_{p \in P} \frac{1}{p^{1+i t+\varepsilon}}=\log Z(1+i t+\varepsilon) \quad \text { et } \quad \ell(t)=\lim _{\varepsilon \rightarrow 0} \ell_{\varepsilon}(t) .
$$

Au voisinage de 0 , on peut écrire

$$
\ell(t)=\log \frac{1}{i t}+\text { fonction analytique }
$$

en convenant que

$$
\log \frac{1}{i t}=\log \frac{1}{|t|}-i \frac{\pi}{2} \operatorname{sign} t
$$

Soit $f \in \mathcal{D}(\mathbb{R})$ et

$$
\widehat{f}(x)=\int f(t) e^{-i t x} \mathrm{~d} t .
$$


Alors

$$
\int f(t) \ell_{\varepsilon}(t) \mathrm{d} t=\sum_{p \in P} \frac{1}{p^{1+\varepsilon}} \widehat{f}(\log p)
$$

et, en faisant tendre $\varepsilon$ vers 0 ,

$$
\int f(t) \ell(t) \mathrm{d} t=\sum_{p \in P} \frac{1}{p} \widehat{f}(\log p) .
$$

Cette formule peut s'appliquer de bien de manières. Montrons d'abord comment l'utiliser pour montrer que $Z(s)$ (donc aussi $\zeta(s)$ ) n'a pas de zéro sur la droite $\sigma=1$.

Si $Z(s)$ avait des zéros sur la droite $\sigma=1$, ils seraient symétriques par rapport à la droite réelle. Désignons alors deux d'entre eux par $1+i t_{0}$ et $1-i t_{0}$, par $k$ leur multiplicité et par $\varphi$ une fonction appartenant à $\mathcal{D}(\mathbb{R})$, de type positif $(\widehat{\varphi} \geq 0)$, telle que $\varphi\left(t_{0}\right)=\varphi\left(-t_{0}\right)>(1 / 2) \varphi(0)$ et nulle sur les zéros de $Z(1+i t)$ à l'exception de $t_{0}$ et $-t_{0}$. Appliquons (1) à $f(t)=\varphi(t) e^{i t y}$, où $y>0$ sera choisi plus tard. Le premier membre est alors la valeur en $y$ de la transformée de Fourier de la fonction $\varphi(t) \ell(t)$, qui ne diffère de la fonction

$$
\varphi(t)\left(\log \frac{1}{i t}-k \log \frac{1}{i\left(t-t_{0}\right)}-k \log \frac{1}{i\left(t+t_{0}\right)}\right)
$$

que par une fonction $\in \mathcal{D}(\mathbb{R})$. Donc ce premier membre vaut

$$
\frac{2 \pi}{y}\left(\varphi(0)-k \varphi\left(t_{0}\right) e^{i t_{0} y}-k \varphi\left(-t_{0}\right) e^{-i t_{0} y}\right)+\mathrm{O}\left(\frac{1}{y^{2}}\right) \quad(y-\infty) .
$$

En choisissant $y$ multiple de $2 \pi / t_{0}$ et grand, sa partie réelle est négative. Cependant, comme $\widehat{f} \geq 0$, le second membre est positif. La contradiction établit que $Z(s)$ n'a pas de zéro sur la droite $\sigma=1$.

Choisissons maintenant $\varphi \in \mathcal{D}(\mathbb{R})$, paire, et $f=\varphi f_{0}$ avec

$$
\widehat{f_{0}}(x)= \begin{cases}e^{x-y} & \text { pour } x \leq y \\ 0 & \text { pour } x>y\end{cases}
$$

La formule (1) donne

$$
\int \frac{\ell(t) \varphi(t)}{1+i t} e^{i t y} \mathrm{~d} t=\int \widehat{\varphi}(x) e^{x-y} \varpi\left(e^{y-x}\right) \mathrm{d} x
$$




\section{Jean-Pierre Kahane}

et, pour la méme raison que ci-dessus, le premier membre vaut

$$
\frac{2 \pi}{y} \varphi(0)+\mathrm{O}\left(\frac{1}{y^{2}}\right) \quad(y \rightarrow \infty) .
$$

On choisit $\varepsilon>0$ et $\varphi$ telle que $\widehat{\varphi} \geq 0, \varphi(0)=1$, et

$$
1-\varepsilon \leq \frac{1}{2 \pi} \int_{-\varepsilon}^{\varepsilon} \widehat{\varphi}(x) \mathrm{d} x\left(<\frac{1}{2 \pi} \int \widehat{\varphi}(x) \mathrm{d} x=1\right) .
$$

Dans ces conditions

$$
\frac{1}{2 \pi} \int \widehat{\varphi}(x) e^{x-y} \varpi\left(e^{y-x}\right) \mathrm{d} x=\frac{1}{y}+\mathrm{O}\left(\frac{1}{y^{2}}\right) \quad(y \rightarrow \infty) .
$$

On minore le premier membre en intégrant sur $[-\varepsilon, \varepsilon]$ et, en faisant tendre $\varepsilon$ vers 0 , on obtient

$$
\varlimsup_{y \rightarrow \infty} y e^{-y} \varpi\left(e^{y}\right) \leq 1 \text {. }
$$

En conséquence

$$
e^{x-y} \varpi\left(e^{y-x}\right) \leq \frac{K}{y-x+1} \quad(x<y)
$$

pour une constante $K$ convenable. On utilise cette majoration sous la forme

$$
e^{x-y} \varpi\left(e^{y-x}\right) \leq \frac{K}{y}
$$

quand $-\infty<x<-\varepsilon$, et sous la forme

$$
\begin{aligned}
e^{x-y} \varpi\left(e^{y-x}\right) & \leq K\left(\frac{1}{y-\varepsilon+1}+\frac{x-\varepsilon}{y-\varepsilon}\left(1-\frac{1}{y-\varepsilon+1}\right)\right) \\
& \leq \frac{K}{y-\varepsilon}(1+x)
\end{aligned}
$$

quand $\varepsilon<x<y$. En choisissant $\varphi$ de manière que

$$
\int_{\varepsilon}^{\infty} \hat{\varphi}(x)(1+x) \mathrm{d} x=\mathrm{o}(1) \quad(\varepsilon-0)
$$

et en majorant le premier membre de (4) en intégrant successivement sur $(-\infty,-\varepsilon) .(-\varepsilon, \varepsilon)$ et $(\varepsilon, y)$, on obtient

$$
\varliminf_{y \rightarrow \infty} y e^{-y} \varpi\left(e^{y}\right) \geq 1,
$$


d'où finalement

$$
\lim _{y \rightarrow \infty} y e^{-y} \varpi\left(e^{y}\right)=1
$$

la conclusion annoncée.

Voici une variante, pour obtenir $\theta(x) \sim x$. On choisit $f=\varphi f_{1}$ avec

$$
\widehat{f}_{1}(x)= \begin{cases}x e^{x-y} & \text { pour } x \leq y \\ 0 & \text { pour } x>y\end{cases}
$$

On obtient

$$
\int \frac{\ell(t) \varphi(t)}{(1+i t)^{2}} e^{i t y} \mathrm{~d} t=\int \widehat{\varphi}(x) e^{x-y} \theta\left(e^{y-x}\right) \mathrm{d} x
$$

et on procède de la même façon.

On peut beaucoup affaiblir la condition (0). En effet, la preuve est presque inchangée si, au voisinage de 0 ,

$$
\ell(t)=\log \frac{1}{i t}+\text { fonction de classe } C^{1}
$$

au lieu de "fonction analytique" (cela amène à remplacer $\mathrm{O}\left(1 / y^{2}\right)$ par o $(1 / y)$ dans les formules (2), (3) et (4)). Or, pour qu'il en soit ainsi, il suffit que

$$
\zeta(1+i t)=\frac{D}{i t}+\text { fonction de classe } C^{1} .
$$

Il suffit donc que

$$
\int_{e}^{\infty}|\nu(x)-D x| \frac{\log x}{x^{2}} \mathrm{~d} x<\infty
$$

pour avoir les conclusions

$$
\varpi(x) \sim \frac{x}{\log x}, \quad \theta(x) \sim x \quad(x \rightarrow \infty) .
$$

Sous la condition encore plus faible

$$
\int_{e}^{\infty}|\nu(x)-D x| \frac{\mathrm{d} x}{x^{2}}<\infty
$$

on aura

$$
\zeta(1+i t)=\frac{D}{i t}+\text { fonction de classe } A,
$$


$A$ désignant l'algèbre de Wiener $\mathcal{F} L^{1}$. Le théorème de Wiener-Lévy permet de passer aux logarithmes et d'obtenir

$$
\ell(t)=\log \frac{1}{i t}+\text { fonction de classe } A_{\text {loc }}
$$

au voisinage de 0 . Si $\zeta(1+i t)$ admet un zéro d'ordre $k>1 / 2$ en $t_{0}$, la transformée de Fourier de $\ell(t) \varphi(t)$ est la somme du premier terme de (2) et de la transformée de Fourier d'une fonction de classe $A$ à support compact, qui est nécessairement $o(1 / y)$ pour une infinité de valeurs de $y$ dans toute progression arithmétique infinie donnée. Or on peut, dans le premier membre de $(2)$, choisir $\varphi\left(t_{0}\right)$ et $\varphi\left(-t_{0}\right)$ arbitrairement proches de 1 (ce n'est ni tout à fait évident, ni difficile à montrer). Donc, sous la condition (6), $\zeta(1+i t)$ n'admet aucun zéro d'ordre $>1 / 2$.

THÉORÈME .- Sous l'hypothèse

$$
\int_{e}^{\infty}\left(\frac{|\nu(x)-D x| \log x}{x}\right)^{2-\varepsilon} \frac{\mathrm{d} x}{x}<\infty
$$

pour un $\varepsilon>0$, on a la conclusion

$$
\varpi(x) \sim \frac{x}{\log x}, \quad \theta(x) \sim x \quad(x \rightarrow \infty) .
$$

Voici l'idée de la preuve. L'hypothèse entraîne que tous les zéros de $\zeta(1+i t)$ sont d'ordre $>1 / 2$ donc, compte tenu de l'alinéa qui précède que $\zeta(1+i t)$ ne s'annule pas. Elle entraîne d'autre part (même quand $\varepsilon=0$ ) que $\zeta(1+i t)$ est localement sur $\mathbb{R} \backslash\{0\}$ une fonction de la classe $H^{1}$ de Sobolev, et la preuve se déroule comme pour la classe $C^{1}$. Les détails seront publiés ailleurs.

L'hypothèse (7) est vérifiée quand

$$
\nu(x)=D x+\mathrm{O}\left((\log x)^{-a}\right) \quad(x \rightarrow \infty)
$$

avec $a>3 / 2$, qui est l'hypothèse de Beurling. D'autre part, un exemple de Beurling, adapté au cas qui nous occupe par Diamond [Di], montre que l'hypothèse

$$
\nu(x)=D x+\mathrm{O}\left((\log x)^{-3 / 2}\right) \quad(x-\infty)
$$

n'entraine pas la conclusion. Cela laisse ouverte la question : suffit-il, pour la conclusion, d'avoir l'hypothèse (7) avec $\varepsilon=0$ ? 


\section{Intermède historique.}

\section{La loi des grands nombres, le lemme de Kronecker, et le théorème 1 de la deuxième note de Stieltjes}

Dans les cours de probabilité on est amené à démontrer la loi des grands nombres

$$
\frac{X_{1}+X_{2}+\cdots+X_{n}}{n} \underset{n \rightarrow \infty}{\longrightarrow} E(X) \text { presque sûrement }
$$

sous la seule hypothèse que $X_{1}, X_{2}, \ldots, X_{n}, \ldots$ est une suite de variables aléatoires indépendantes, de même distribution, intégrables et d'espérance $E(X)$. Quitte à soustraire $E(X)$, on peut supposer $E(X)=0$. Une démonstration classique consiste à définir une suite $Y_{n}$ telle que p.s. $X_{n}=$ $Y_{n}$ pour $n$ grand et à établir d'abord que la série $\sum Y_{n} / n$ converge presque sûrement, puis à utiliser un "lemme de Kronecker" selon lequel la convergence d'une série $\sum a_{n} / n$ entraîne que $\left(a_{1}+\cdots+a_{n}\right) / n$ tend vers zéro.

Or le théorème 1 de la seconde note de Stieltjes énonce que, pour tout $\sigma>0$, la convergence de la série $\sum a_{n} / n^{\sigma}$ entraîne que $\left(a_{1}+\cdots+a_{n}\right) / n^{\sigma}$ tend vers zéro. Cela suffit pour la loi des grands nombres $(\sigma=1)$ et pour ses variantes $(\sigma>1)$.

Le "lemme de Kronecker" est un énoncé un peu plus général, qui rend la preuve assez transparente : si la série $\Sigma \alpha_{n}$ converge et si $\beta_{n}$ est une suite positive croissante tendant vers l'infini,

$$
\lim _{n \rightarrow \infty} \frac{\alpha_{1} \beta_{1}+\alpha_{2} \beta_{2}+\cdots+a_{n} \beta_{n}}{\beta_{n}}=0 .
$$

Il se trouve généralement énoncé sans référence, et comme Kronecker (18231891) a publié l'essentiel de ses ouvres avant 1885 , on peut se demander si le lemme de Kronecker n'est pas largement antérieur à l'énoncé de Stieltjes.

Ce n'est pas le cas. Kronecker n'a publié son résultat qu'en 1886, dans une note aux Comptes rendus, présentée par Hermite, dans laquelle il se réfère explicitement à Stieltjes. Il est intéressant de lire sa conclusion. Après avoir, dans le dernier paragraphe de sa note, donné des théorèmes d'interversion de limites, avec application à l'estimation de la somme des logarithmes des nombres premiers compris entre deux bornes, il écrit : 
"J'ai déjà donné plusieurs fois les développements contenus dans ce dernier paragraphe, dans les leçons que je professe à l'université sur l'application de l'Analyse à la Théorie des nombres; ils se trouvent, en particulier, dans le cours du semestre d'hiver 1875-1876 qui a été rédigé par M. Hettner, actuellement professeur à Berlin. Mais c'est par un article de M. Stieltjes [suit en note la référence à la note du 3 août 1885], auquel la Science doit déjà plusieurs Mémoires très intéressants, que j'ai été porté à rédiger cette note, après en avoir communiqué les points principaux a mon ami M. Hermite au commencement de septembre 1885. " [K]

Rappelons le rôle du théorème 1 dans la note de Stieltjes. Supposant établi que la série

$$
\sum \frac{1-\Lambda(n)}{n^{\sigma}}
$$

converge sur $\sigma>3 / 4$, le théorème 1 permet d'écrire

$$
\sum_{n<x}(1-\Lambda(n))=\mathrm{o}\left(x^{3 / 4+\varepsilon}\right) \quad(x \rightarrow \infty)
$$

pour tout $\varepsilon>0$, d'où $\theta(x)=x+\mathrm{o}\left(x^{3 / 4+\varepsilon}\right)(x \rightarrow \infty)$.

\section{Le théorème 2 de la deuxième note et sa postérité : convergence des séries produits}

La formule

$$
\left(\sum_{1}^{\infty} \frac{a_{n}}{n^{s}}\right)\left(\sum_{1}^{\infty} \frac{b_{n}}{n^{s}}\right)=\sum_{1}^{\infty} \frac{c_{n}}{n^{s}}
$$

définit formellement la série du second membre, $C$, quand sont données formellement les séries du premier membre, $A$ et $B$. Les coefficients $c_{n}$ sont fournis par

$$
c_{n}=\sum_{m \mid n} a_{m} b_{n / m}
$$

et $C$ s'appelle la série produit de $A$ et de $B$. Il est immédiat que si, en un point donné $s, A$ et $B$ convergent absolument, il en est de même pour $C$. Si $A$ est convergente et $B$ absolument convergente, $C$ est convergente; c'est facile à montrer et énoncé par Stieltjes dans son article de 1887. Le théorème 2 de la deuxième note de Stieltjes en 1885 est plus subtil. 
$\left(\mathrm{S}_{0}\right)$ Si $A$ et $B$ sont convergentes pour $s=\alpha$ et absolument convergentes pour $s=\alpha+\beta$ ( $\alpha$ réel, $\beta \geq 0), C$ est convergente pour $s=$ $\alpha+(1 / 2) \beta$.

Rappelons l'application qu'en fait Stieltjes aux séries

$$
\begin{aligned}
& A: \sum \frac{d(n)-\log n-2 C}{n^{s}}\left(=\zeta^{2}+\zeta^{\prime}-2 C \zeta\right) \\
& B=\sum \frac{\mu(n)}{n^{s}}\left(=\frac{1}{\zeta}\right) .
\end{aligned}
$$

Les deux sont absolument convergentes pour $s>1$, et Stieltjes les croit convergentes pour $s>1 / 2$. C'est vrai pour $A$, et la convergence pour $A$ a même lieu dès que $s>1 / 3$ (Voronoï 1903; voir Landau III [L2, p. 100]); c'est encore en question pour $B$, et le mieux qu'on sache est la convergence en $s=1$ (van Mangolt 1897; voir Landau III [L2, p. 331] et [He]).

En 1887, Stieltjes indiqua, sans démonstration, deux variantes que j'énoncerai avec les notations utilisées plus tard par Landau.

$\left(\mathrm{S}_{1}\right) \mathrm{Si} A$ et $B$ sont convergentes pour $s=\rho$ et absolument convergentes respectivement pour $s=\rho+\tau$ et $s=\rho+\tau^{\prime}, C$ est convergente pour $s=\rho+\tau \tau^{\prime} /\left(\tau+\tau^{\prime}\right)$.

$\left(\mathrm{S}_{2}\right) \mathrm{Si} A$ et $B$ sont convergentes pour $s=\rho, C$ est convergente pour $s=\rho+1 / 2$.

$\left(\mathrm{S}_{1}\right)$ est une généralisation de $\left(\mathrm{S}_{0}\right)$. Sous l'hypothèse de $\left(\mathrm{S}_{2}\right)$, les séries $A$ et $B$ sont absolument convergentes quand $s>\rho+1$, et il résulte de $\left(\mathrm{S}_{0}\right)$ que $C$ est convergente lorsque $s>\rho+1 / 2$. On voit que $\left(\mathrm{S}_{2}\right)$ apporte un complément : $C$ est convergente lorsque $s \geq \rho+1 / 2$.

Le rôle de cette translation par $1 / 2$ n'est pas évident. Dans sa thèse en 1894 , Cahen énonça que, sous les hypothèses de $\left(\mathrm{S}_{2}\right), C$ converge dès que $s>\rho$. C'est une erreur, relevée par Landau dans son monumental article de 1907 sur la multiplication des séries de Dirichlet (Landau III [L1, p. 362]) : il existe un $h>0$ et deux séries $A$ et $B$, convergentes en 0 , telles que la série $C$ soit divergente en $h$ [L1].

Dans ce même article Landau démontre des généralisations de $\left(S_{1}\right)$ et $\left(\mathrm{S}_{2}\right)$, que nous désignerons par $\left(\mathrm{L}_{1}\right)$ et $\left(\mathrm{L}_{2}\right)$. 
$\left(\mathrm{L}_{1}\right)$ Supposons $A$ convergente en $\rho$ et absolument convergente en $\rho+\tau, B$ convergente en $\rho^{\prime}$ et absolument convergente en $\rho^{\prime}+\tau^{\prime}$, avec $\tau \geq 0$, $\tau^{\prime} \geq 0, \tau+\tau^{\prime}>0, \rho^{\prime} \leq \rho+\tau, \rho \leq \rho^{\prime}+\tau^{\prime}$. Alors $C$ converge au point

$$
\sigma=\frac{\rho \tau^{\prime}+\rho^{\prime} \tau+\tau \tau^{\prime}}{\tau+\tau^{\prime}}
$$

Les hypothèses $\rho^{\prime} \leq \rho+\tau$ et $\rho \leq \rho^{\prime}+\tau^{\prime}$ nécessitent un commentaire. Si l'une d'elles, par exemple la première, est violée, $A$ est absolument convergente au point $\rho^{\prime}$ où l'on suppose la convergence de $B$; on voit alors que $C$ converge en $\rho^{\prime}$ et on ne peut rien dire de mieux.

$\left(\mathrm{L}_{2}\right)$ Supposons $A$ convergente en $\rho$ et $B$ convergente en $\rho^{\prime}$, avec $\left|\rho-\rho^{\prime}\right|<1$. Alors $C$ est convergente en $\sigma=(1 / 2)\left(\rho+\rho^{\prime}+1\right)$.

La condition sur $\rho$ et $\rho^{\prime}$ peut faire l'objet du méme commentaire que ci-dessus.

Nous allons voir que $\left(\mathrm{S}_{1}\right),\left(\mathrm{S}_{2}\right),\left(\mathrm{L}_{1}\right)$ et $\left(\mathrm{L}_{2}\right)$ sont inaméliorables en ce sens que l'on ne peut pas diminuer la valeur de $s$ ou $\sigma$ donnée en conclusion. Pour $\left(S_{2}\right)$, c'est un résultat de la thèse de $B$ ohr; pour $\left(S_{1}\right),\left(L_{1}\right)$ et $\left(L_{2}\right)$ cela résulte d'une variante de la méthode de Bohr que j'exposerai rapidement dans la suite (Kahane et Queffélec 1995 [KQ]).

Cependant on peut prolonger ces résultats de deux manières. La première consiste à introduire une sommabilité liée à un paramètre au lieu de la convergence. C'est ce que Bohr a fait en 1950 [B3], et j'indiquerai à la fin de cet article comment on peut étendre sa méthode et ses résultats. On peut aussi considérer un produit de plus de deux facteurs. Le cas des puissances $k^{\text {ièmes }}$ a été indiqué dans un article à la mémoire de $\mathrm{H}$. Bohr [Ka1, p. 109]. Un cas plus général, avec un énoncé plus précis, a été étudié par Delange et Tenenbaum [DT]. Nous allons exposer les résultats de Kahane et Queffélec, qui contiennent tous les précédents [KQ].

Il nous faut d'abord exposer et exploiter la méthode par laquelle Bohr a montré que la conclusion de $\left(\mathrm{S}_{2}\right)$ est inaméliorable. 


\section{La fonction de Lindelöf et la méthode de Bohr.} Son extension grâce aux catégories de Baire. Résultats optimaux sur la convergence d'une série produit de séries de Dirichlet

Une fonction analytique $f(s)$ définie dans une bande verticale $a<\sigma<b$ ( $s=\sigma+i t)$ y est dite d'ordre fini si elle y est majorée par un polynôme en $t$. La fonction d'ordre, ou fonction de Lindelöf, est

$$
\mu(\sigma)=\inf \left\{\alpha \geq 0\left|f(\sigma+i t)=\mathrm{O}\left(|t|^{\alpha}\right),\right| t \mid \rightarrow \infty\right\} .
$$

C'est une fonction convexe (Lindelöf 1908). Si $f(s)$ est la somme d'une série de Dirichlet ou son prolongement analytique dans le demi-plan $\sigma>a, \mu(\sigma)$ est décroissante, nulle pour $\sigma=\sigma_{a}$ (abscisse de convergence absolue), $\leq 1$ pour $\sigma=\sigma_{c}$ (abscisse de convergence, cela résulte du travail de pionnier de Jensen en 1884) et $\sigma_{c} \leq \omega_{\mu}$, la plus petite valeur de $\sigma$ pour laquelle $\mu(\sigma)=0$ (Schnee 1908) [HR]. Dans sa thèse (1910), Bohr construit une série de Dirichlet pour laquelle $\sigma_{a}=1, \sigma_{c}=0$ et $\mu(\sigma)=(1-\sigma)^{+}$(partie positive de $1-\sigma)$ sur $(0, \infty)$. Soit $A$ cette série de Dirichlet. La fonction de Lindelöf de son carré $A^{2}$ est $(2-2 \sigma)^{+}$, donc, si la série $A^{2}$ converge en un point $\sigma$, on a $2-2 \sigma \leq 1$, soit $\sigma \geq 1 / 2$. D'après $\left(\mathrm{S}_{2}\right)$ (avec $B=A, \rho=0$ ) la série $C=A^{2}$ est convergente en $\sigma=1 / 2$, mais elle n'est convergente pour aucun $\sigma<1 / 2$ [B1].

L'usage de cette fonction de Bohr peut s'étendre un peu; par exemple, si la série $A^{k}(k$ entier $\geq 1)$ converge en $\sigma$, on a $k-k \sigma \leq 1$, soit $\sigma \geq 1-1 / k$, et cela laisse pressentir quel peut être le résultat optimal pour la convergence d'une puissance $k^{\text {ième }}$ d'une série de Dirichlet convergente en zéro ([Ka1], $[\mathrm{DT}])$.

Cependant, pour montrer que les conclusions que $\left(\mathrm{S}_{1}\right),\left(\mathrm{L}_{1}\right),\left(\mathrm{L}_{2}\right)$, et a fortiori que les conclusions de leurs généralisations à des produits de plus de deux facteurs sont optimales, la seule fonction de Bohr ne suffit pas. On a besoin du théorème suivant.

THÉORÈmE [KQ]. - Étant donnés des $\rho_{j}$ réels et des $0<\tau_{j} \leq 1, j=$ $1,2, \ldots, k$, il existe pour chaque $j$ une série de Dirichlet $A_{j}$, convergente en $\rho_{j}$, absolument convergente en $\rho_{j}+\tau_{j}$, dont la fonction de Lindelöf est

$$
\mu_{j}(\sigma)=\frac{1}{\tau_{j}}\left(\rho_{j}+\tau_{j}-\sigma\right)^{+}
$$


pour $\sigma \geq \rho_{j}$, avec la propriété supplémentaire que la fonction de Lindelöf de tout produit partiel des $A_{j}$ est égale à la somme des $\mu_{j}(\sigma)$ correspondantes :

$$
C=\prod_{j \in J} A_{j} \Longrightarrow \mu_{c}(\sigma)=\sum_{j \in J} \mu_{j}(\sigma) \quad \text { pour } \sigma>\sup _{J} \rho_{j}
$$

La démonstration du théorème serait très laborieuse en s'inspirant directement de la construction de Bohr. Elle est au contraire très accessible en utilisant des séries de la forme

$$
\sum_{n} \varepsilon_{j, n}\left(\lambda_{j, n}^{-\left(s-\rho_{\jmath}\right)}-\mu_{j, n}^{-\left(s-\rho_{\jmath}\right)}\right)
$$

avec

$$
\varepsilon_{j, n}= \pm 1, \quad \lambda_{j, n}=\left[(2 n-1)^{-\left(1 / \tau_{\jmath}\right)}\right], \quad \mu_{j, n}=\left[(2 n)^{-\left(1 / \tau_{\jmath}\right)}\right] .
$$

L'ensemble des $\varepsilon_{j, n}$, soit $\Omega$, est un espace de Baire. Sur un $G_{\xi}$ dense (nous dirons : quasi-sùrement sur $\Omega$ ), les séries écrites possèdent toutes les propriétés requises pour les $A_{j}$ dans le théorème.

Corollaire 1. - Étant donnés des $\rho_{j}$ réels et des $0<\tau_{j} \leq 1$, $j=1,2, \ldots, k$, soit $\sigma$ la solution de l'équation

$$
\sum_{1}^{k} \frac{1}{\tau_{j}}\left(\rho_{j}+\tau_{j}-\sigma\right)^{+}=1
$$

(qui est $\geq \sup \rho_{j}$ ). Il existe des séries de Dirichlet $A_{j}$ telles que chaque $A_{j}$ soit convergente en $\rho_{j}$ et absolument convergente en $\rho_{j}+\tau_{j}$, et que le produit $C=A_{1} A_{2} \cdots A_{k}$ soit divergent en tout point strictement à gauche de $\sigma$.

Une variante consiste à donner les $\rho_{j}$ mais non les $\tau_{j}$. Il existe alors pour chaque $j$ une série de Dirichlet $A_{j}$ convergente en $\rho_{j}$ dont la fonction de Lindelöf est

$$
\mu_{j}(\sigma)=\left(\rho_{j}+1-\sigma\right)^{+}
$$

pour $\sigma \geq \rho_{j}$, avec la propriété supplémentaire énoncée dans le théorème. 
Corollaire 2.- Si l'on associe aux $\rho_{j}$ la solution $\sigma$ de

$$
\sum_{1}^{k}\left(\rho_{j}+1-\sigma\right)^{+}=1,
$$

on obtient des séries de Dirichlet convergentes aux points $\rho_{j}$, dont le produit est divergent strictement à gauche de $\sigma$.

Pour $k=2$, on voit que les estimations de $s$ et de $\sigma$ données dans $\left(\mathrm{S}_{1}\right)$, $\left(\mathrm{L}_{1}\right)$ et $\left(\mathrm{L}_{2}\right)$ sont inaméliorables. En effet, sous les hypothèses de $\left(\mathrm{L}_{1}\right),\left(\mathrm{C}_{1}\right)$ s'écrit

$$
\frac{1}{\tau}(\rho+\tau-\sigma)+\frac{1}{\tau^{\prime}}\left(\rho^{\prime}+\tau^{\prime}-\sigma\right)=1
$$

soit

$$
\sigma=\frac{\rho \tau^{\prime}+\rho^{\prime} \tau+\tau \tau^{\prime}}{\tau+\tau^{\prime}}
$$

et de même, sous les hypothèses de $\left(\mathrm{L}_{2}\right),\left(\mathrm{C}_{2}\right)$ s'écrit

$$
(\rho+1-\sigma)+\left(\rho^{\prime}+1-\sigma\right)=1
$$

soit $\sigma=(1 / 2)\left(\rho+\rho^{\prime}+1\right)$.

Mais $\left(\mathrm{C}_{1}\right)$ et $\left(\mathrm{C}_{2}\right)$ suggèrent des énoncés qui font disparaitre les restrictions sur $\rho$ et $\rho^{\prime}$ dans les hypothèses, à savoir :

$\left(\mathrm{L}_{1}^{\prime}\right)$ si $A$ est convergente en $\rho$ et absolument convergente en $\rho+\tau$, $B$ convergente en $\rho^{\prime}$ et absolument convergente en $\rho^{\prime}+\tau^{\prime}, C$ est convergente en $\sigma$, donné par $\left(\mathrm{C}_{1}\right)$;

$\left(\mathrm{L}_{2}^{\prime}\right)$ si $A$ est convergente en $\rho$ et $B$ convergente en $\rho^{\prime}, C$ est convergente en $\sigma$, donné par $\left(\mathrm{C}_{2}\right)$.

$\left(\mathrm{L}_{1}^{\prime}\right)$ est correct, et c'est le meilleur résultat possible, mais $\left(\mathrm{L}_{2}^{\prime}\right)$ ne l'est pas : on a un contre-exemple en choisissant

$$
A=\sum \frac{(-1)^{n}}{n^{s}} a_{n}, \quad B=\sum \frac{(-1)^{n}}{n^{s+1}} \beta_{n},
$$

$a_{n}$ et $\beta_{n}$ décroissant très lentement vers zéro, de façon que $A$ converge en 0 et $B$ en -1 , mais $C$ diverge en 0 . Il s'avère que c'est essentiellement le seul cas d'exception.

$\left(\mathrm{L}_{2}^{\prime}\right)$ est correct sous la seule condition que $\left|\rho-\rho^{\prime}\right| \neq 1$, et c'est le meilleur résultat possible. 
Dans le cas d'un produit de $k$ facteurs on a les résultats suivants.

$\left(\mathrm{KQ}_{1}\right) .-$ On considère $k$ séries de Dirichlet $A_{1}, A_{2}, \ldots, A_{k}$, et on suppose que $A_{j}$ est convergente en $\rho_{j}$ et absolument convergente en $\rho_{j}+\tau_{j}$, avec $0<\tau_{j} \leq 1, j=1,2, \ldots, k$. Soit $\sigma$ la solution de l'équation $\left(C_{1}\right)$. Alors la série $C=A_{1} A_{2} \cdots A_{k}$ est convergente en $\sigma$, et c'est le meilleur résultat possible.

$\left(\mathrm{KQ}_{2}\right)$. - On considère $k$ séries de Dirichlet $A_{1}, A_{2}, \ldots, A_{k}$, et on suppose que $A_{j}$ convergente en $\rho_{j}, j=1,2, \ldots, k$. Soit $\sigma$ la solution de l'équation $\left(C_{2}\right)$ et supposons $\rho_{1} \leq \rho_{2} \leq \ldots \leq \rho_{k}$. Alors la série $C=A_{1} A_{2} \cdots A_{k}$ est convergente $\grave{a}$ droite (strictement) de $\sigma$ dans tous les cas, et aussi au point $\sigma$ si $\sigma \neq \rho_{j}+1$ pour tout $j$, et c'est le meilleur résultat possible hors ce cas d'exception.

Dans l'énoncé $\left(\mathrm{KQ}_{1}\right)$ on a supposé $\tau_{j}>0$ pour tout $j$ pour pouvoir utiliser l'équation $\left(\mathrm{C}_{1}\right)$. Si l'on suppose seulement $\tau_{j} \geq 0$ l'énoncé s'adapte immédiatement : si tous les $\tau_{j}$ sont nuls, on prend pour $\sigma$ le maximum des $\rho_{j}$; sinon, on réduit la somme dans $\left(\mathrm{C}_{1}\right)$ aux $j$ tels que $\tau_{j}>0$ et on prend pour $\sigma$ le maximum de la racine de $\left(\mathrm{C}_{1}\right)$ et des $\rho_{j}$ pour lesquels $\tau_{j}=0$.

On peut aussi préciser l'ensemble des $s$ complexes pour lesquels la convergence de la série $C$ est assurée. C'est en général le demi-plan fermé $\Re s \geq \sigma$ (donné par $\left(\mathrm{C}_{1}\right)$ ou $\left.\left(\mathrm{C}_{2}\right)\right)$, et il $\mathrm{y}$ a des exceptions lorsque $\rho_{k} \geq \sup _{j \neq k}\left(\rho_{j}+\tau_{j}\right)\left(\operatorname{cas}\left(\mathrm{KQ}_{1}\right)\right)$ ou $\rho_{k} \geq \rho_{k-1}+1\left(\operatorname{cas}\left(\mathrm{KQ}_{2}\right)\right)$.

Cela contient comme cas particulier le théorème de Delange et Tenenbaum, relatif au cas $(k-1)\left(\rho_{k}-\rho_{1}\right)<1$. Dans ce cas, et plus généralement lorsque $\sum_{j=1}^{k}\left(\rho_{j}-\rho_{1}\right)<1$, la racine de $\left(\mathrm{C}_{2}\right)$ est

$$
\sigma=\frac{1}{k}\left(\rho_{1}+\rho_{2}+\cdots+\rho_{k}\right)+1-\frac{1}{k} .
$$

\section{Ordre et sommabilité. Théorème de Cesàro. Théorème de Bohr. Définitions de Marcel Riesz. Problèmes et conjectures}

Les méthodes de sommabilité de Cesàro apparaissent en 1890, dans un article consacré à la multiplication des séries de Taylor considérées au point $z=1$. Ici donc 


$$
\begin{gathered}
A(z)=\sum_{0}^{\infty} a_{n} z^{n}, \quad B(z)=\sum_{1}^{\infty} b_{n} z^{n}, \\
C(z)=A(z) B(z)=\sum_{1}^{\infty} c_{n} z^{n}, \quad c_{n}=\sum_{m} a_{m} b_{n-m},
\end{gathered}
$$

et $A, B, C$ divisent les séries $A(1), B(1), C(1)$. Le premier théorème de Cesàro est le suivant : si $A$ et $B$ sont convergentes, $C$ est sommable par le procédé de la moyenne arithmétique. Plus généralement, si $A$ est sommable $(C, \alpha)$ (procédé de Cesàro d'ordre $\alpha$ ) et $B$ sommable $(C, \beta), C$ est sommable $(C, \alpha+\beta+1)$. Cesàro avait défini $(C, \alpha)$ pour $\alpha$ entier. La définition et le théorème furent étendus ensuite à des ordres de sommation réels $>-1$.

Rappelons les définitions, qui rendent le théorème presque évident : $A$ est $(C, \alpha)$-bornée, resp. $(C, \alpha)$-convergente $(=$ sommable $)$ vers zéro, si les coefficients de Taylor du produit $(1-z)^{-\alpha-1} A(z)$ sont grand O, resp. petit $\mathrm{o}$, de ceux de la fonction $(1-z)^{-\alpha-1} ; A$ est $(C, \alpha)$-convergente (= sommable) vers $\ell$ si $A-\ell$ est $(C, \alpha)$-convergente (= sommable) vers zéro.

Bohr et Marcel Riesz ont étendu le théorème de Cesàro aux séries de Dirichlet. De nouveau maintenant $A, B, C$ désignent les séries

$$
\begin{gathered}
\sum_{1}^{\infty} \frac{a_{n}}{n^{s}}, \quad \sum_{1}^{\infty} \frac{b_{n}}{n^{s}}, \quad \sum_{1}^{\infty} \frac{c_{n}}{n^{s}}, \\
c_{n}=\sum_{m \mid n} a_{n} b_{n / m} .
\end{gathered}
$$

Bohr considérait la $(C, \alpha)$-sommabilité en se restreignant aux $\alpha$ entiers. Pour le cas $a>0$, il vaut mieux considérer la sommabilité définie par Riesz, que nous écrivons $(R, \alpha)[\mathrm{HR}]$. On dira qu'au point $s=0$, la série $A$ est $(R, \alpha)$-bornée, resp. $(R, \alpha)$-convergente vers 0 , resp. $(R, \alpha)$-convergente vers $\ell$ si

$$
\begin{array}{ll} 
& \sum_{n<\exp x} a_{n}(x-\log n)^{\alpha}=\mathrm{O}\left(x^{\alpha}\right) \quad(x \rightarrow \infty), \\
\text { resp. } & \sum_{n<\exp x} a_{n}(x-\log n)^{\alpha}=\mathrm{o}\left(x^{\alpha}\right) \quad(x \rightarrow \infty), \\
\text { resp. } & \sum_{n<\exp x}\left(a_{n}-\ell\right)(x-\log n)^{\alpha}=\mathrm{o}\left(x^{\alpha}\right) \quad(x \rightarrow \infty) .
\end{array}
$$


Il revient au même d'écrire, avec les notations familières aux physiciens, en posant $\mu=\sum a_{n} \delta_{\log n}$ (à ne pas confondre avec la fonction $\mu(\sigma)$ de Lindelöf!),

$$
H x^{\alpha} * \mu=\mathrm{O}\left(H x^{\alpha}\right) \quad(x \rightarrow \infty) \text { etc. }
$$

Le fait que la série produit $C$ soit $(R, \alpha+\beta+1)$-bornée, resp. sommable (= convergente), lorsque les séries $A$ et $B$ sont $(R, \alpha)$ et $(R, \beta)$-bornées, resp. sommables, vient de la formule

$$
H x^{\alpha} * H x^{\beta}=C_{\alpha, \beta} H x^{\alpha+\beta+1} .
$$

Toutes les formules de convolution sur $\mathbb{R}^{+}$se transforment en multiplication des transformées de Laplace, et la transformée de Laplace de $\mu$ est précisément la série de Dirichlet $A$.

Bohr et M. Riesz attachent à la série de Dirichlet $A$ les ensembles de couples $(\sigma, \alpha)(\sigma$ réel, $\alpha>0)$ tels qu'au point $s=\sigma$ la série $A$ soit $(C, \alpha)$ ou $(R, \alpha)$-sommables. Ces deux ensembles ont le mème intérieur [HR, pp. 23 et 45 , théorème 30$]$. Nous admettons, comme le fait Bohr, les résultats suivants, qu'il attribue à $\mathrm{M}$. Riesz (une partie figure dans le livre de Hardy et Riesz sans démonstration) : l'intérieur des ensembles ci-dessus est défini par $a>\psi(\sigma)$; sauf si $\psi(\sigma)=\infty$ pour tout $\sigma, \psi$ est une fonction convexe, décroissante, à pente $\leq-1$ quand $\psi(\sigma)>0$, nulle à droite d'une certaine valeur $\omega_{\psi}$. Ainsi

$$
\omega_{\psi}=\inf \{\sigma \mid \psi(\sigma)=0\}, \quad \psi^{\prime}\left(\omega_{\psi}-0\right) \leq-1,
$$

cette inégalité n'ayant de sens, naturellement, que pour $\omega_{\psi}>-\infty$. Nous admettons également la double inégalité de Bohr

$$
\psi(\sigma) \leq \mu(\sigma) \leq \psi(\sigma)+1
$$

où $\mu(\sigma)$ est la fonction de Lindelöf de la série $A$.

Dans son dernier article, posthume, Bohr s'attache à construire des séries de Dirichlet dont on impose les fonctions d'ordre et de sommabilité [Bo4]. Il montre qu'étant donné deux fonctions $\mu(\sigma)$ et $\psi(\sigma)$ vérifiant (B) et vérifiant toutes deux les propriétés signalées plus haut pour $\psi$, il existe une série de Dirichlet les admettant pour fonctions d'ordre et de sommabilité respectivement. Il pose la question : pour la fonction d'ordre $\mu(\sigma)$, a-t-on nécessairement $\psi^{\prime}\left(\omega_{\mu}-0\right) \leq-1$ quand on définit $\omega_{\mu}=\inf \{\sigma \mid \mu(\sigma)=0\}$ ? 
Une réponse positive, observe-t-il, entraînerait tout de suite, pour la série $\sum(-1)^{n} / n^{s}, \mu(\sigma)=(1 / 2-\sigma)^{+}$(l'égalité est connue pour $\sigma \geq 1$ et pour $\sigma \leq 0)$, c'est-à-dire la validité de la conjecture de Lindelöf.

La réponse à cette question de Bohr est négative; on peut construire des séries de Dirichlet telles que $\mu^{\prime}\left(\omega_{\mu}-0\right)$ soit arbitrairement proche de $-1 / 2$. La caractérisation des fonctions d'ordre des séries de Dirichlet reste un problème ouvert [Ka2].

Sur la fonction de sommabilité d'autres questions se posent. On peut d'abord considérer la condition

$$
H x^{\alpha} * \mu=\mathrm{O}\left(H x^{\alpha}\right) \quad(x \rightarrow \infty)
$$

pour une mesure $\mu$ localement bornée sur $\mathbb{R}^{+}$, et pour $\alpha>-1$ : on dira alors que $\mu$ est $(R, \alpha)$-bornée. De façon immédiate, si $\mu$ est $(R, \alpha)$-bornée et $\nu$ est $(R, \beta)$-bornée, $\mu * \nu$ est $(R, a+\beta+1)$-bornée. On peut aussi associer à $\mu$ la fonction de sommabilité.

$$
\psi(\sigma)=\inf \left\{\alpha \mid e^{-\sigma x} \mathrm{~d} \mu(x) \text { est }(R, \alpha) \text {-bornée }\right\} .
$$

Les fonctions $\psi(\sigma)$ sont maintenant à valeurs dans $[-1, \infty]$, et les fonctions $1+\psi(\sigma)$ s'ajoutent quand on convolue des mesures. Il serait bon de montrer que les fonctions $\psi(\sigma)$ sont convexes, et de les caractériser.

Si $\mathrm{d} \mu$ est une mesure discrète, on a $\psi(\sigma) \geq 0$. Mais il existe d'autres définitions de la fonction de sommabilité qui permettent de lui donner $[-\infty, \infty]$ comme domaine des valeurs. On peut pour cela s'inspirer de la première définition que $\mathrm{M}$. Riesz a donné des moyennes typiques en 1909, et des retouches qu'il a apportées en 1924 [R]. Ainsi, si $\mu=\sum_{1}^{\infty} a_{n} \delta_{\lambda_{n}}$ $\left(0<\lambda_{1}<\lambda_{2}<\ldots\right)$, on pourra dire que $\mu$ est $\left(R_{0}, \alpha\right)$-bornée si

$$
\sum_{n=1}^{n-p}\left(\lambda_{n}-\lambda_{m}\right)^{\alpha} a_{n}=\mathrm{O}\left(\lambda_{n}^{\alpha}\right) \quad(n \rightarrow \infty)
$$

pour tout entier $p$ fixé, et définir la nouvelle fonction de sommabilité

$$
\psi_{0}(\sigma)=\inf \left\{\alpha \mid e^{-\sigma x} \mathrm{~d} \mu(x) \text { est }\left(R_{0}, \alpha\right) \text {-bornée }\right\} .
$$

De la sorte, $\psi_{0}(\sigma)$ mesure non seulement le degré de divergence de la série de Dirichlet $\sum a_{n} e^{-\lambda_{n} \sigma}$ pour les petites valeurs de $\sigma$, mais la rapidité de convergence pour les grandes. Il serait bon d'établir que $\psi_{0}(\sigma)$ est une fonction convexe et que c'est un prolongement de $\psi(\sigma)$. Mais les propriétés de $\left(R_{0}, \alpha\right)$ et de $\psi_{0}(\sigma)$ ne sont pas évidentes. 


\section{La sommabilité d'une série produit de séries de Dirichlet}

Le sujet est traité par H. Bohr dans un article de 1950 [B3] pour un produit de deux séries. En général, le problème le plus simple se présente ainsi : on s'intéresse à des séries de Dirichlet ordinaires $\left(\lambda_{n}=\log n\right)$; on suppose que la série $A_{j}$ est $\left(R, \alpha_{j}\right)$-sommable en $0(j=1,2, \ldots, \ldots, k)$. Que peut-on dire de la fonction de sommabilité $\psi_{C}(\sigma)$ du produit $C=$ $A_{1} A_{2} \cdots A_{k}$ ? La réponse est :

$$
\psi_{C}(\sigma)=\left(\sum_{1}^{k}\left(1+\alpha_{j}-\sigma\right)^{+}-1\right)^{+} \quad(\sigma \geq 0)
$$

et c'est le meilleur résultat possible quand les $a_{j}$ sont donnés.

La démonstration se trouvera dans [KQ]. La première étape consiste, en utilisant les techniques quasi-sûres de Queffelec dans [Q], à construire les $A_{j}$ de façon que l'égalité ait lieu dans $(*)$. La seconde, à savoir la preuve de $(*)$, utilise, comme chez Bohr, quatre ingrédients :

1) les propriétés générales des fonctions de sommabilité (convexité, pente $\leq-1$ ) d'où résulte en particulier que

$$
\psi_{A_{j}}(\sigma) \leq\left(\left(1+\alpha_{j}-\sigma\right)^{+}\right)^{+}
$$

$2)$ le fait que si deux séries sont $(R, \alpha)$ et $(R, \beta)$-bornées, leur produit est $(R, \alpha+\beta+1)$-borné;

$3)$ le fait que la $(R, a)$-sommabilité est conservée par multiplication par une série absolument convergente;

4) une version faible de $\left(\mathrm{KQ}_{2}\right)$, à savoir que les hypothèses $\psi_{A_{j}}\left(\alpha_{j}\right)=0$ entraînent $\psi_{C}(\sigma)=0$ dès que le second membre de $(*)$ est nul.

On opère une récurrence sur $k$ en ordonnant les $a_{j}$ dans l'ordre décroissant, et on distingue les cas $\sum_{j}\left(\alpha_{j}-\alpha_{k}\right)<1$ et $\geq 1$. Dans le premier cas, on utilse 1),2) et 3 ) et dans le second 1),2) et 4). 


\section{Références}

[Ba] BANG (T.) .- An inequality for real functions of a real variable and its application to the prime number theorem, Proc. Oberwolfach Conference on Approximation theory 1963, pp. 155-160.

[Be] Beurling (A.) . - Analyse de la loi asymptotique de la distribution des nombres premiers généralisés, Acta Math. 68 (1937), pp. 255-291; Collected works II, pp. 1-37.

[B1] Bohr (H.) .- Bidrag til de Dirichlet'ske Rackkers Theori, Thèse, Copenhague 1910 (IIIS 1 en traduction anglaise dans les Collected mathematical works, C.M.W.).

[B2] Bohr (H.) .- Zur Theorie der Dirichletschen Reihen, Math. Zeitschrift 52 (1950), pp. 709-722 (C.M.W. I A 18).

[B3] BOHR (H.) .- On multiplication of summable Dirichlet series, Math. Tidsskrift B (1950), pp. 71-75 (C.M.W. I A 19).

[B4] BoHR (H.) .- On the summability function and the order function of Dirichlet series, Det Kgl. Danske Videnskabernes Selskab. Matematisk-Fysiske Meddelelser 27, n $^{\circ} 4$ (1952), pp. 1-39 (C.M.W. I A 22).

[C] Ces̀̀ro (E.) . - Sur la multiplication des séries, Bull. Sc. Math. 14. nº 2 (1890), pp. 114-120.

[Da] Daboussi (H.) .- - Sur le théorème des nombres premiers, C.-R. Acad. Sci. 298 (1984), pp. 161-164.

[DT] Delange (H.) et Tenenbaum (G.) .- Un théorème sur les séries de Dirichlet, Monatsch. für Math. 113 (1992), pp. 99-105.

[Di] Diamond (H. G.) .- A set of generalized numbers showing Beurling's theorem to be sharp, Ill. J. Math. 14 (1970), pp. 29-34.

[EM] Ellison (W. J.) et Mendès-France (M.) - - Les nombres premiers, Paris, Hermann 1975 .

[E] ERDös (P.) .- On a new method in elementary number theory [...], Proc. Nat. Acad. Sci. 35 (1949), pp. 374-384.

[Ha] Hadamard (J.) .- Sur la distribution des zéros de la fonction $\zeta(s)$ et ses conséquences arithmétiques. Bull. Sci. Math. France 14 (1896), pp. 199-220.

[HR] HARDY (G. H.) et RiEsz (M.) .- The general theory of Dirichlet's series, Cambridge tracts in math. and math. physics 18 (1915).

[He] Helson (H.) .- Convergence of Dirichlet series. L'analyse harmonique dans le domaine complexe, Montpellier 1972, Springer Lect. Notes Math. 336 (1973), pp. 153-160.

[I] Ingham (A. E.) - - The distribution of prime numbers, Cambridge tracts in math. and math. physics 30 (1932), (1990).

[Ka1] Kahane (J.-P.) .- Around Bohr's thesis, Proc. Symp. Copenhagen (April 1987) on the Harald Bohr Centenary, Matematisk-fysiske Meddelelser 42, $\mathrm{n}^{\circ} 3$ (1989), pp. 103-111. 
[Ka2] Kahane (J.-P.) . - The last problem of Harald Bohr, J. Austral. Math. Soc. A 47 (1989), pp. 133-152.

[KQ] Kahane (J.-P.) et QuefFÉlec (H.) .- Order, convergence et sommabilité de produits de séries de Dirichlet, Prépublication Orsay, 1995.

[K] KRONECKer (L.) .- Quelques remarques sur la détermination des valeurs moyennes, C.-R. Acad. Sci. 103 (1886), pp. 980-987.

[L1] LANDAU (E.) .- Über die Multiplikation Dirichlet'scher Reihen, Rendiconti del Circ. Matem. Palermo 24 (1907), pp. 81-160.

[L2] Landau (E.) .- Handbuck der Lehre von der Verteilung der Primzahlen, Teubner, Leipzig 1909.

[N] Newman (D. J.) . - Simple analytic proof of the prime number theorem, Amer. Math. Monthly 87 (1980), pp. 693-696.

[Q] QuefFelec (H.) .- Propriétés presque sûres et quasi- sûres des séries de Dirichlet et des produits d'Euler, Canad. J. Math. 32 (1980), pp. 531-558.

[R] Riesz (M.) .- Sur l'équivalence de certaines méthodes de sommation, Proc. London Math. Soc. 22, $\mathrm{n}^{\circ} 2$ (1924), pp. 412-419.

[Se] Selberg (A.) .- An elementary proof of the prime number theorem, Ann. of Math. 50 (1949), pp. 305-313.

[S1] Stieltjes (T. J.) . - Sur une fonction uniforme C.-R. Acad. Sci. 101 (1885), pp. 153-154.

[S2] STIELT JEs (T. J.) . - Sur une loi asymptotique dans la théorie des nombres, C.-R. Acad. Sci. 101 (1885), pp. 368-370.

[S3] Stieltjes (T. J.) . - Note sur la multiplication de deux séries, Nouv. Ann. Math 6, $\mathrm{n}^{\circ} 3(1887)$, pp. 210-215.

[S4] Stieltjes (T. J.) .- Euvres complètes, Collected papers I, II, edited by Gerrit van Dijk. Springer-Verlag 1993.

[VP] VALLÉE-POUSSIN (CH. DE LA) - - Recherches analytiques sur la théorie des nombres, 1, Ann. Soc. Scient. Bruxelles 20 (1896), pp. 183-256.

[W] Wiener (N.) .- Tauberian theorems, Ann. of Math. 33 (1932), pp. 1-100.

[Wi] Wirsing (E.) .- Elementare Beweise des Primzahlsatzes mit Restglied, I, J. für die reine und angew. Math. 211 (1962), pp. 205-219. 